\title{
Visual field constriction associated with vigabatrin: retinal nerve fiber layer photographic correlation
}

A n 18 year old male who had been taking vigabatrin for complex partial seizure was referred for visual field testing. The cumulative vigabatrin dose was about $6.0 \mathrm{~kg}$ over the seven and a half year period. There were bilateral symmetric visual field constrictions with greater loss of the nasal field (fig 1). On the retinal nerve fibre layer (RNFL) photographs discernible RNFL losses in the peripheral retina with a relative preservation of papillomacular bundles were observed bilaterally (fig 2). RNFL thickness around the optic disc, as measured by optical coherence tomography, was markedly reduced in all quadrants except for the temporal quadrant in both eyes and the inferior quadrant in the right eye (fig 3).

Vigabatrin inhibits irreversibly $\gamma$ aminobutyric acid (GABA) transaminase, resulting in an increase in GABA in the brain and in the retina. ${ }^{1}$ As a result, irreversible visual field constriction may be developed. ${ }^{2}$ The mechanism of visual dysfunction is poorly understood but an altered inner retinal function with ganglion cell loss may be a possible cause. ${ }^{23}$ Pathological study at postmortem supported the idea that the primary site of injury lies within the ganglion cells of the retina. ${ }^{4}$

In the present case, RNFL loss was demonstrated by RNFL photography. This finding correlated with the visual field defect and quantitative analysis of RNFL thickness. Further study is necessary in patients taking vigabatrin to determine whether RNFL photography identify RNFL loss before visual field defect development. If it does, as it does in glaucoma, RNFL photography will be a valuable tool for deciding whether to continue with vigabatrin administration.
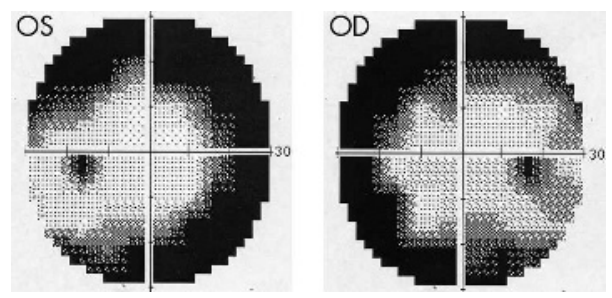

Figure 1 Central 30-2 threshold test using a Humphrey field analyzer (Zeiss, Dublin, California, USA) showed bilateral symmetric visual field constrictions with a greater loss in the nasal visual field (OD: right eye; OS: left eye)
H J Choi, D M Kim

Department of Ophthalmology, Seoul National University Hospital, Seoul, Korea

Correspondence to: Dr D M Kim, Department of Ophthalmology, Seoul National University Hospital, 28 Yongon-Dong, Chongno-Gu, Seoul 110744, Korea; dmkim@snu.ac.kr

\section{References}

1 Schechter PJ, Tranier Y, Jung MJ, et al. Audiogenic seizure protection by elevated brain GABA concentration in mice: effects of gamma-acetylenic GABA and gamma-vinyl GABA, two irreversible GABA-T inhibitors. Eur J Pharmacol 1977;45:319-28.

2 Lawden MC, Eke T, Degg C, et al. Visual field defects associated with vigabatrin therapy. J Neurol Neurosurg Psychiatry 1999;67:716-22.

3 Daneshvar H, Racette L, Coupland SG, et al. Symptomatic and asymptomatic visual loss in patients taking vigabatrin. Ophthalmology 1999;106:1792-8.

4 Ravindran J, Blumbergs $\mathrm{P}$, Crompton J, et al. Visual field loss associated with vigabatrin: pathological correlations. J Neurol Neurosurg Psychiatry 2001;70:787-9

5 Sommer A, Katz J, Quigley HA, et al. Clinically detectable nerve fiber atrophy precedes the onset of glaucomatous field loss. Arch Ophthalmol 1991; 109:77-83.
OD

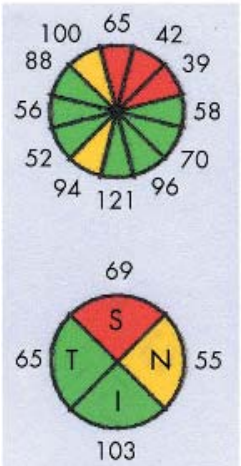

OS

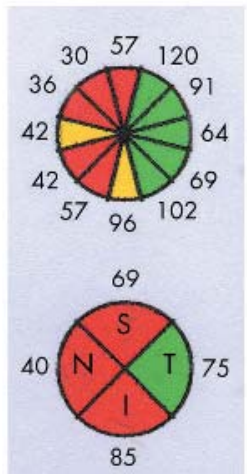

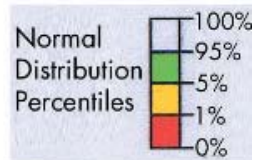

Figure 3 RNFL thickness $(\mu \mathrm{m})$ around the optic disc measured by optical coherence tomography (STRATUS ${ }^{T M}$, Zeiss, Dublin, California, USA) was markedly reduced in all quadrants except for the temporal quadrant in both eyes and the inferior quadrant in the right eye. The red colour indicates that the RNFL thickness of the quadrant is less than in $1 \%$ of normal population and the yellow colour indicates less than in $5 \%$ (S: superior; I: inferior; $\mathrm{N}$ : nasal; T: temporal) (OD: right eye; OS: left eye)
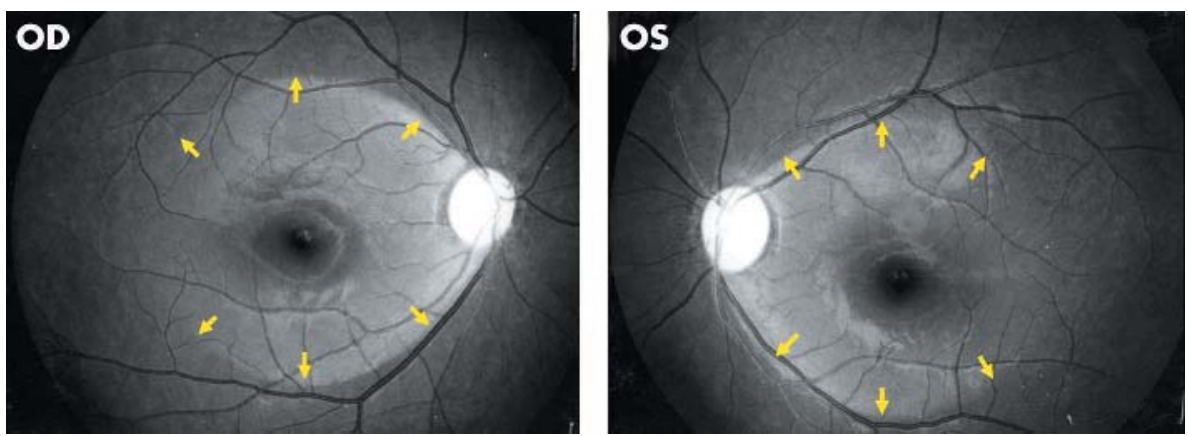

Figure 2 Retinal nerve fiber layer (RNFL) photography using a fundus camera (CF-60 UVi, Canon,

Utsunomiya, Japan) showed discernible RNFL losses in the peripheral retina and a relative preservation of papillomacular bundles in both eyes. Arrows indicate the demarcation between lost and preserved RNFL. 